\title{
Mechanical properties of in situ composites based on polycarbonate and a liquid crystalline polymer
}

\author{
Qinghuang Lin* and Albert F. Yee \\ Department of Materials Science and Engineering, University of Michigan, Ann Arbor, \\ MI 48109-2136. USA \\ (Received 22 November 1993; revised 10 January 1994)
}

\begin{abstract}
The mechanical properties of in situ composites based on blends of polycarbonate and a liquid crystalline copoly(ester amide) (Vectra B950) have been studied as functions of the liquid crystalline polymer (LCP) concentration and the draw ratio, a processing parameter. It is shown that both the elastic modulus and the tensile strength of the in situ composites increase steadily with the LCP concentration and the draw ratio. However, the ultimate tensile strain decreases with these two parameters. A model is proposed for the longitudinal elastic modulus of the in situ composites, which is based on the Halpin-Tsai equation and Northolt's model for the LCP phase. The experimental elastic moduli of the in situ composites are found to conform fairly well with the theoretical values derived from the model.
\end{abstract}

(Keywords: in situ composites; liquid crystalline polymer; polycarbonate)

\section{INTRODUCTION}

Blending two or more polymers is an effective way to combine the useful properties of each component into one system. Recently, polymer blends of liquid crystalline polymers (LCPs) with isotropic polymers have attracted considerable attention because of the potential for producing high performance polymeric materials at reduced costs. Addition of a LCP to an isotropic polymer not only enhances its mechanical properties, due to the easy molecular orientation of the LCP, but also improves its processing properties, as a result of the low melt viscosity of the LCP. On the other hand, combining a LCP with a conventional isotropic polymer is expected to improve the weak lateral strength of the LCP and also to enhance the compressive resistance of the oriented polymer. Moreover, addition of conventional, isotropic thermoplastics also lowers the cost of the final products.

Another important advantage of polymer blends containing LCPs is that addition of the latter makes it possible to control blend morphology through the processing. Under the appropriate processing conditions, the dispersed LCP phase can be deformed into a fibrillar phase, which acts as the reinforcing phase. The resultant product is hence called an in situ composite ${ }^{1}$. The deformation of the LCP phase into the fibrillar form not only increases the aspect ratio of the reinforcing phase, but also improves the molecular orientation of the LCP chains. As a consequence, significant reinforcement can be achieved with the addition of only a small amount of LCP ${ }^{1-13}$. The in situ composite concept has emerged as a new route for the development of advanced polymeric materials.

* To whom correspondence should be addressed
The key to successful fabrication of in situ composites is the formation of a fibrillar LCP morphology. To generate this type of morphology, three major methods have been used, namely fibre spinning, extrusion, and injection molding. It has been found that fibre spinning is particularly advantageous because the elongational force field in the spinning process is more effective for producing the LCP fibrillar morphology than the shear force field that is present in other processing methods.

As in other multiphase polymer systems, the mechanical properties of in situ composites depend on the composition, the properties of each component, the macro- and microstructures of the composites, and on the interfacial adhesion and compatibilization. In particular, the shape and dispersion of the LCP phase and the orientation of the LCP chains play primary roles in determining the mechanical properties. Furthermore, the overall properties of the composites are dominated by the properties of the reinforcing LCP phase, since the mechanical properties of the LCP fibrils are typical of those of high performance fibres. It has been well established that a fibrillar LCP morphology translates into higher moduli and strengths. The reinforcement effect arises from the larger aspect ratio of the fibrillar LCP phase and the better molecular orientation of the LCP chains ${ }^{5,7,9}$.

Previous studies of the mechanical properties of in situ composites focused mainly on the effects of LCP concentration, certain processing conditions, such as drawing, and compatibilization. Addition of a LCP to an isotropic polymer generally leads to an increase in modulus and strength, and a decrease in ultimate tensile strain. Improvements in modulus and strength, which range from tens of percent to an order of magnitude, 
have been found in blends of LCPs and isotropic thermoplastics, such as polycarbonate ${ }^{1,4-9,11,14-22}$, polystyrene $e^{3,13,17,23}$, polyamides (nylon) ${ }^{1,2,14,24,25}$, polyethylene terephthalate) ${ }^{17,26-30}$, poly(butylene terephthalate $^{1,14}$, polypropylene ${ }^{12,27,31}$, poly(ether imide $)^{1,8,10,32}$, poly(ether ether ketone $)^{1,33}$, poly(ether sulfone $)^{1}$, polysulfone $^{34}$, poly(phenyl sulfide $)^{35}$, poly(arylate) $)^{1,36}$, and the blends of some of these polymers ${ }^{13,22,37,38}$. Drawing leads to additional improvements in mechanical properties, e.g. both modulus and strength have been found to increase with drawing. The improvements in the mechanical properties are generally related to a fibrillar LCP morphology which is produced by the elongational stress field during the drawing process. Recently, Bretas and Baird ${ }^{38}$ demonstrated that compatibilization of the immiscible in situ composites of polypropylene and a liquid crystalline copoly(ester amide) (Vectra) also results in an impressive improvement in mechanical properties. However, there are instances in which no reinforcement was achieved with addition of a LCP to either polypropylene or poly(butylene terephthalate) ${ }^{31,39}$.

We have been interested in developing these novel self-reinforced in situ composites, using a liquid crystalline copoly(ester amide) as the discrete LCP phase and polycarbonate or polystyrene as the continuous phase $\mathrm{e}^{3-7}$. We used the fibre spinning method to generate the selfreinforced composite structure. Our previous results showed that the addition of only $30 \mathrm{wt} \% \mathrm{LCP}$ to polycarbonate leads to a 7.3 times increase in elastic modulus and a 1.4 times increase in tensile strength, relative to the corresponding properties of pure polycarbonate ${ }^{\text {. }}$.

The purpose of this paper is to present a systematic study of the mechanical properties of the in situ composites based on polycarbonate and a thermotropic copoly(ester amide) (Vectra B950). Specifically, the effects of the LCP concentration and the blend draw ratio on tensile modulus, tensile strength and ultimate tensile strain are investigated. In addition, a model is proposed for the elastic modulus of the in situ composites, which is based on the Halpin-Tsai equation and Northolt's model for the LCP phase. Theoretical predictions of the model are then compared with the experimental results obtained for the longitudinal elastic modulus of the in situ composites.

\section{EXPERIMENTAL}

\section{Materials}

The LCP used in this research was Vectra B950 (Hoechst Celanese). Vectra B950 is a random liquid crystalline copoly(ester amide) of three components: $60 \mathrm{~mol} \%$ 2,6-hydroxynaphthoic acid, $20 \mathrm{~mol} \%$ terephthalic acid and $20 \mathrm{~mol} \%$-aminophenol. The isotropic thermoplastic matrix that was used was a bisphenol-A polycarbonate (PC) (Lexan 151, GE Plastics).

\section{Processing}

In situ composite fibres were fabricated by melt extrusion of blends of the LCP and polycarbonate with a ZSK-30 twin-screw extruder (Werner and Pfleiderer). The extrusion was followed by drawing the extruded melt directly with a take-up machine, with the draw ratio being varied by changing the speed of the take-up reels. The detailed procedures are described elsewhere ${ }^{7}$. Three series of samples were fabricated: one varied in LCP content but had a constant draw ratio of $\sim 11.5$, while the other two varied in draw ratio but had constant LCP concentrations of 20 and $26.9 \mathrm{vol} \%$, respectively. The draw ratio was determined experimentally from the ratio of the cross-sectional area of the die to that of the extruded composite fibre.

\section{Mechanical testing}

The longitudinal elastic moduli of the extruded fibres were measured on an Instron Mechanical Tester (Model 4502), while the elongation was determined with the aid of an extensometer attached directly to the specimen. To reduce stress concentration at the grips, the specimens were held in place with rubber pads. Since the strain was measured directly over a predetermined gauge section, the displacement in the grips did not affect the accuracy of the results. All tests were run at a constant crosshead speed of $0.5 \mathrm{~mm} \mathrm{~min}^{-1}$. All data were acquired and processed by an IBM computer, with each data point reported here being the average of five measurements.

\section{RESULTS AND DISCUSSION}

\section{Stress-strain behaviour}

Typical stress-strain curves of the in situ composite are shown in Figure 1. We note that, in contrast to pure polycarbonate, most drawn composite fibres do not exhibit any yielding during tensile testing. Only those with low LCP contents and low draw ratios show limited yielding and stress whitening.

\section{Effect of melt drawing}

One of the advantages of in situ composites is the possibility of improving the mechanical properties by controlling the blend morphology through processing. Previous studies demonstrated that melt drawing, which usually generates fibrillar LCP morphologies, led to enhancements in modulus and strength in many of the in situ composite systems ${ }^{7-11,18,24,25,36,39,40}$. However, other researchers ${ }^{13,20,32.37}$ have reported that drawing did not appreciably change either the modulus or the strength. In some instances, drawing was even reported to have a detrimental effect on the mechanical properties $^{13,37}$.

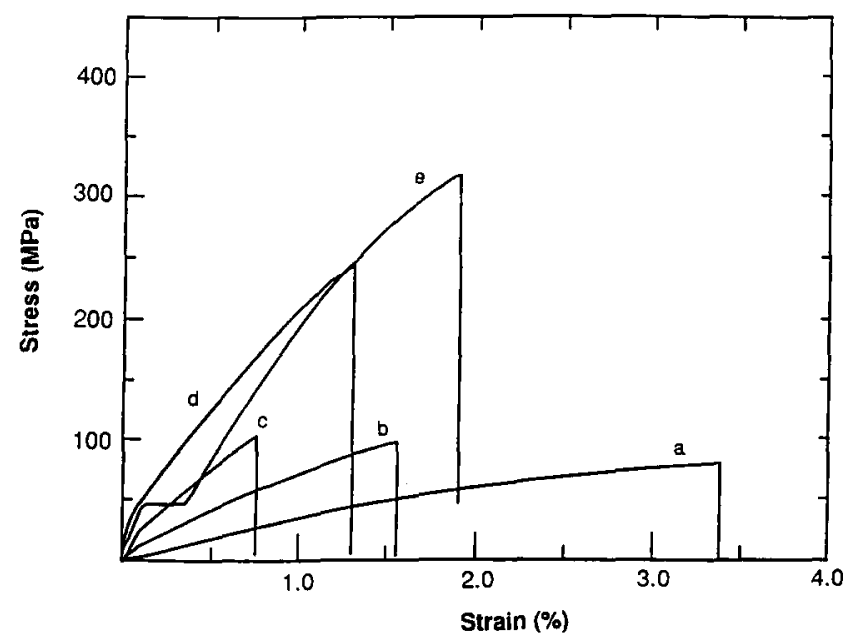

Figure 1 Typical stress-strain curves of PC/LCP in situ composites with different LCP contents: (a) $10 \mathrm{vol} \%$; (b) $20 \mathrm{vol} \%$; (c) $26.9 \mathrm{vol} \%$; (d) $57.3 \mathrm{vol} \%$; (e) $77.4 \mathrm{vol} \%$. The draw ratio for all samples was 11.5 


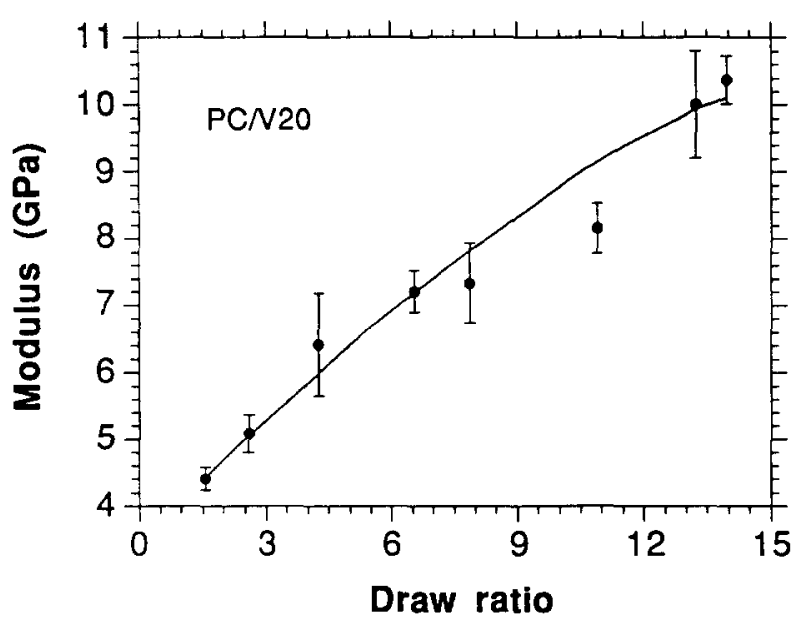

Figure 2 Effect of draw ratio on elastic modulus of PC/LCP in situ composites with $20 \mathrm{vol} \%$ LCP

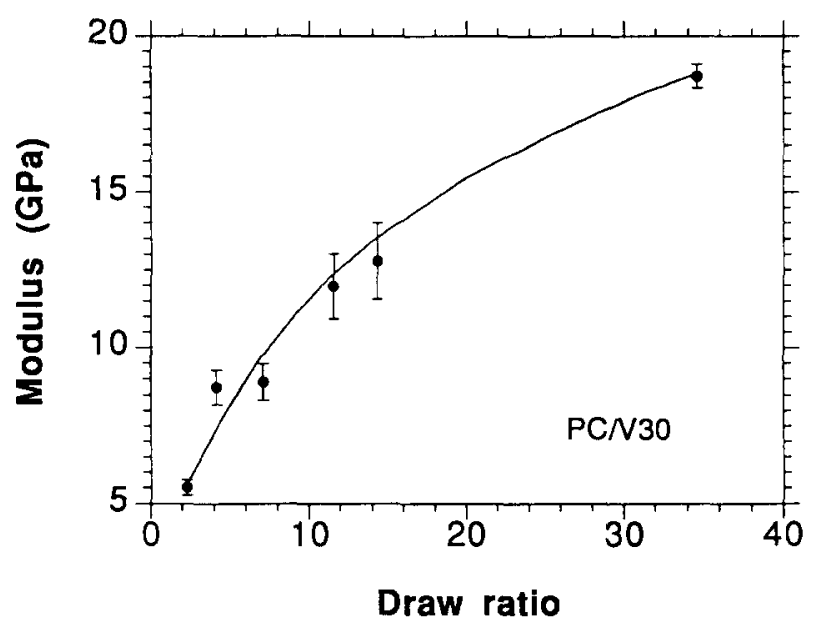

Figure 3 Effect of draw ratio on elastic modulus of PC/LCP in situ composites with 26.9 vol\% LCP

We have found that drawing the extruded strands results in marked improvements in the mechanical properties. Figures 2-5 show the effect of drawing on the elastic modulus and tensile strength of the in situ composites containing 20 and 26.9 vol \% LCP. It is seen that both the elastic modulus and the tensile strength increase monotonically with the draw ratio. Increasing the draw ratio from 1.6 to 14 leads to a $136 \%$ improvement in the modulus and a $67 \%$ improvement in the tensile strength for the composite with $20 \mathrm{vol} \%$ LCP. With higher draw ratios, the improvement for the composites with $26.9 \mathrm{vol} \% \mathrm{LCP}$ is even more significant. For example, the elastic modulus of the composites with a draw ratio of 35 is more than three times that of an undrawn specimen. This finding is in agreement with results previously reported for most of the $\mathrm{LCP} /$ isotropic polymer systems. The apparent drop in the tensile strength of the composite with $26.9 \mathrm{vol} \% \mathrm{LCP}$ at the highest draw ratio is due to a change in the gripping method that was used during the mechanical testing (see Figure 5) ${ }^{7}$.

Our previous studies have demonstrated that the application of a stress field through drawing brings about two major structural changes in the blends ${ }^{7}$ :

(1) With increasing draw ratio, the dispersed LCP phase is deformed from a spherical form, first into an ellipsoidal, and then into a fibrillar form. In other words, the aspect ratio of the dispersed LCP phase is increased during drawing.

(2) The (semi)rigid LCP chains are oriented along the fibre axial direction during drawing. X-ray scattering measurements of the blends indeed showed a steady increase in the order parameter of the LCP chains with draw ratio ${ }^{5}$. Both of these structural changes could lead to improvements in the elastic modulus and tensile strength of the composites.

During the drawing process, some molecular orientation of the polycarbonate phase may result. However, mechanical measurements of a number of isotropic polymers showed that melt drawing does not appreciably alter the modulus and the strength of the isotropic polymers ${ }^{3,18,20,32,36,37,40}$. This may be due to the flexible nature of these isotropic polymer chains and/or the rapid relaxation of their molecular orientation. It is much more difficult to extend and orient flexible chains than rigid chains, because the former involves a huge penalty in the entropy. Therefore, the structural change of the polycarbonate during drawing will not have an appreciable influence on the overall mechanical properties of the in situ composites. This is also true because of the fact that the mechanical properties of the blends are dominated by the reinforcing LCP phase.

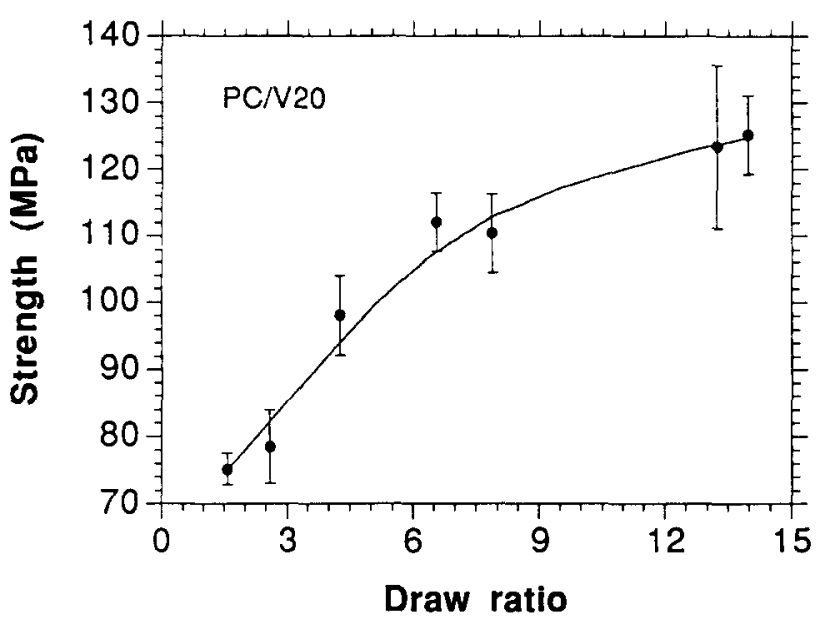

Figure 4 Effect of draw ratio on tensile strength of PC/LCP in situ composites with 20 vol $\%$ LCP

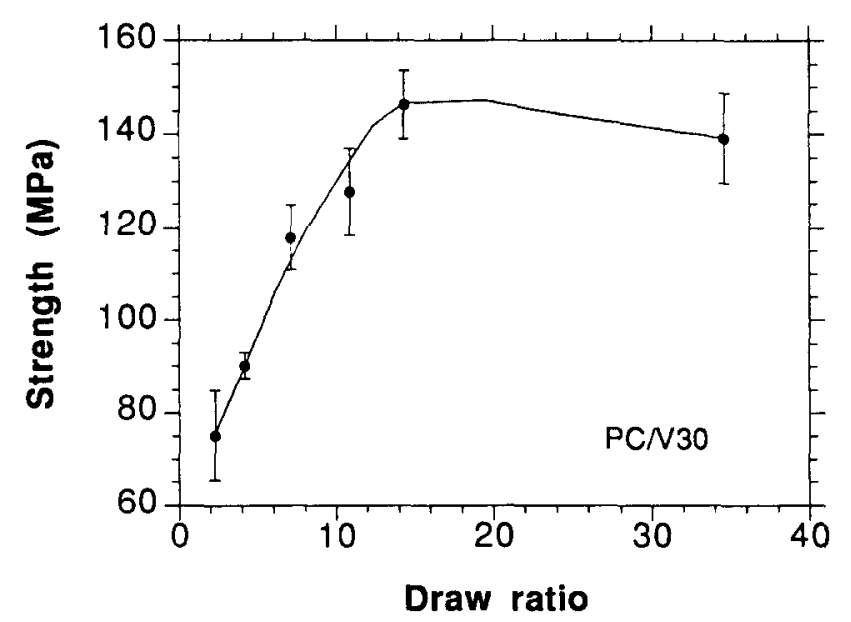

Figure 5 Effect of draw ratio on tensile strength of PC/LCP in situ composites with $26.9 \mathrm{vol} \%$ LCP 


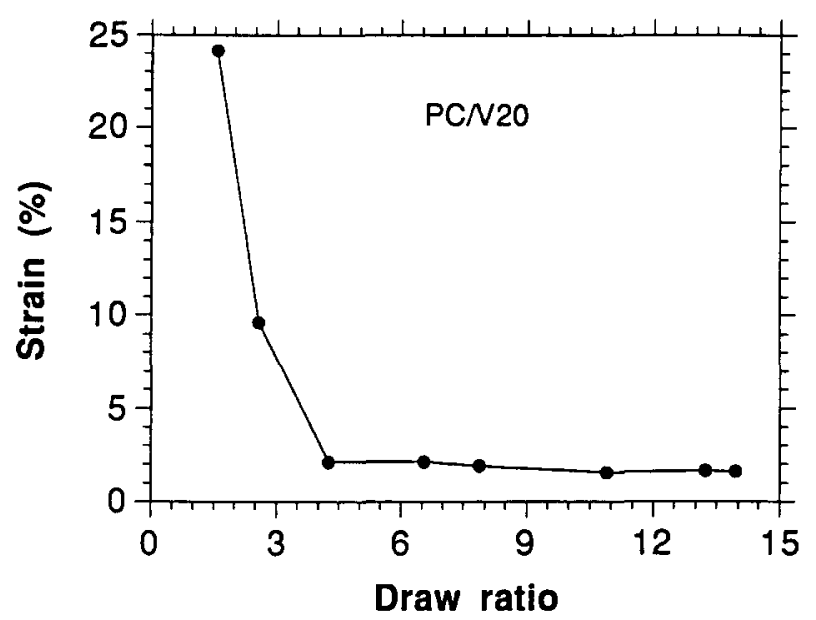

Figure 6 Effect of draw ratio on ultimate tensile strain of PC/LCP in situ composites with $20 \mathrm{vol} \%$ LCP

Figure 6 gives the variation of ultimate tensile strain with the draw ratio for the in situ composites containing $20 \mathrm{vol} \%$ LCP. It is clearly shown that the ultimate tensile strain decreases dramatically with drawing, with this behaviour beginning at the lower draw ratios. It then levels off at a draw ratio of $\sim 4$. Similar behaviour has previously been observed in other in situ composite systems, but no explanation, however, has been offered in these cases $^{10,13,25}$.

\section{Effect of LCP concentration}

According to the published literature, the effect of LCP concentration on the mechanical properties of in situ composites is more complicated than the effect of melt drawing. Most of the published papers have shown reinforcement with the addition of a LCP to an isotropic polymer, although the magnitude of the reinforcement and the dependence on LCP content $\operatorname{vary}^{1,2,4,6,8,11-16,18,20,21,23-28,30,35,36,38}$. We and many other groups ${ }^{1,4,7-14}$ have found steady improvements in modulus and strength with increasing LCP concentration. However, Crevecoeur and Groeninckx ${ }^{13}$ and others ${ }^{19,23}$ found that the reinforcement effect resulted only after the LCP concentration exceeded certain values in injection moulded samples. Li et al. ${ }^{26}$ observed that the dependence of elastic modulus and tensile strength on the LCP content followed two distinct paths as the LCP changed from the discrete phase to the continuous phase in drawn composite fibres. Isayev and Subramanian ${ }^{33}$ noted maxima and minima in modulus and strength as the LCP concentration was increased. Finally, Ajji et $a l^{39}$ reported that the mechanical properties did not change with the addition of up to $30 \mathrm{wt} \%$ LCP to poly(butylene terephthalate).

We found steady increases in elastic modulus and tensile strength with the LCP content in the polycarbonate/ Vectra in situ composites. The results are depicted in Figures 7 and 8 for samples with an average draw ratio of 11.5. It is seen that reinforcement can be achieved throughout the whole concentration range studied, from 10 up to $\sim 80$ vol\% LCP. This is in good agreement with most of the previous studies on other LCP/isotropic polymer systems. The reason appears to be our ability to consistently generate fibrillar morphologies as long as the LCP remains as the discrete phase. Aside from the LCP volume effect, X-ray measurements showed that the molecular orientation of the LCP chains increases slightly with increasing LCP concentration ${ }^{41}$. It is interesting to note that both the elastic modulus and the tensile strength follow a similar trend with increasing LCP content without any abrupt deviation, even though phase inversion occurs in the vicinity of $60 \mathrm{vol} \% \mathrm{LCP}$ in the $\mathrm{LCP} /$ polycarbonate system.

Figure 9 gives the variation of ultimate tensile strain versus LCP concentration at an average draw ratio of 11.5. It is noted that the ultimate tensile strain decreases with the LCP content at lower LCP concentrations, but increases slightly at higher LCP concentrations, with the minimum strain being located at a LCP concentration of $\sim 30 \mathrm{vol} \%$. The reason for the existence of the minimum ultimate tensile strain has not yet been completely understood at this point, although it appears to be related to the change in morphology of the blends. A continuous polycarbonate phase was observed for blends with LCP concentrations below $30 \mathrm{vol} \%$, while a continuous LCP phase was seen for blends with LCP concentrations above $60 \mathrm{vol} \%$.

A model for the elastic modulus of in situ composites

The extensive experimental work on the mechanical properties of in situ composites has prompted researchers to model these properties. While modelling tensile

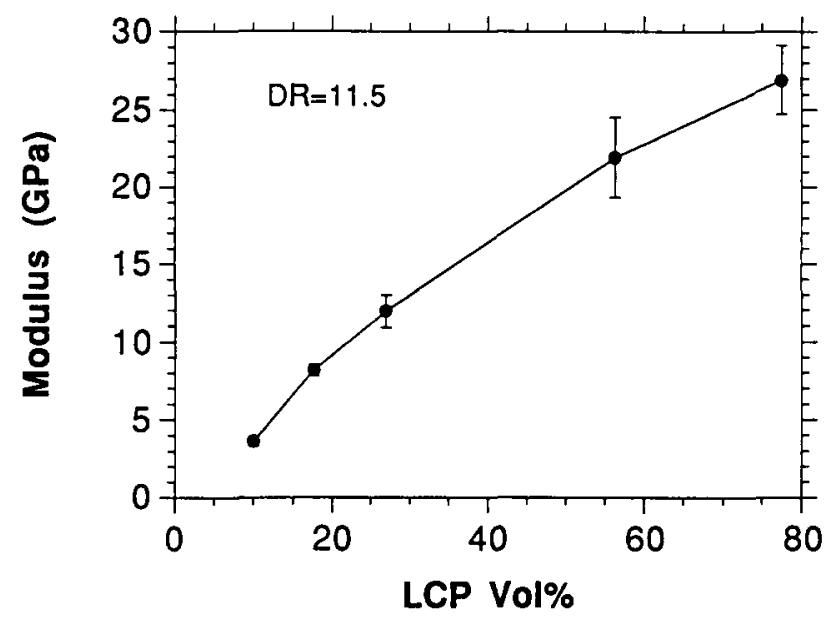

Figure 7 Effect of LCP concentration on elastic modulus of PC/LCP in situ composites with a draw ratio of 11.5

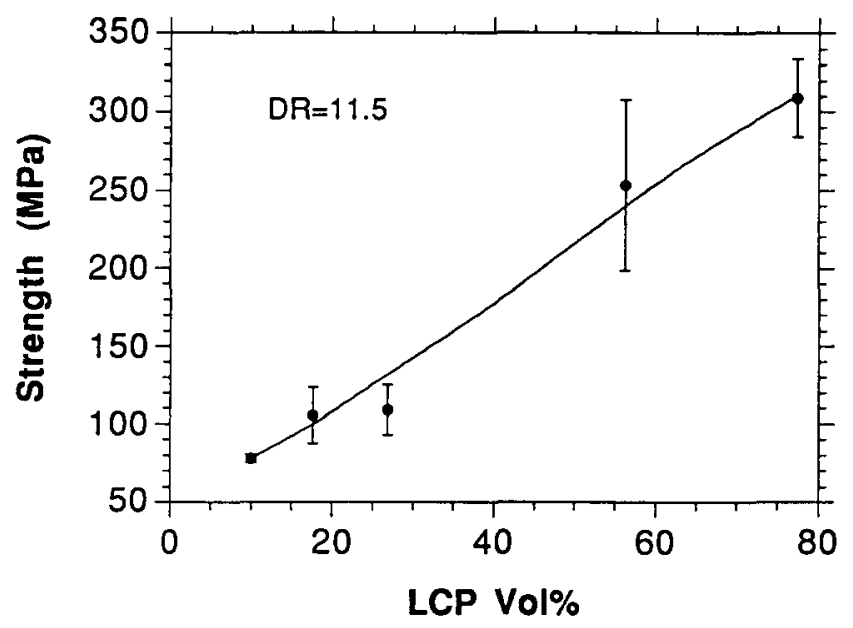

Figure 8 Effect of LCP concentration on tensile strength of PC/LCP in situ composites with a draw ratio of 11.5 


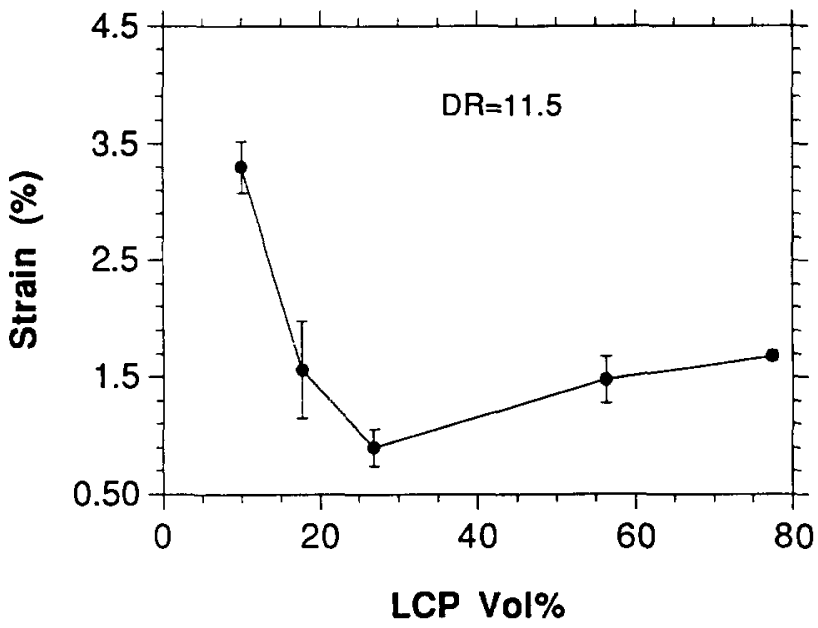

Figure 9 Effect of LCP concentration on ultimate tensile strain of PC/LCP in situ composites with a draw ratio of 11.5

strength is difficult, attempts have been made to apply the simple rule of mixtures of continuous fibre-reinforced composites to modelling the modulus of the in situ composites, even though in most cases the morphologies of the in situ composites were far from that of continuous fibre-reinforced composites ${ }^{3,4,8,13,15,16,22,26,32,33,35,37}$. Recently, some researchers ${ }^{13,26,29}$ have indicated that the aspect ratio of the LCP reinforcing phase could seriously affect the elastic modulus of the in situ composites. However, even in these cases, constant elastic moduli were assumed for the reinforcing LCP microfibrils. This assumption is not reasonable because the molecular orientation of the LCP reinforcing phase increases with drawing, and the elastic modulus of the LCP microfibrils is expected to change accordingly. In fact, a careful examination of the literature revealed that the values used for the elastic modulus of the reinforcing LCP fibrils (Vectra A) ranged from $2.2 \mathrm{GPa}^{22}$ to $75 \mathrm{GPa}^{13}$ with many intermediate values, e.g. $10 \mathrm{GPa}^{35}, 24.6 \mathrm{GPa}^{8}$, $26.4 \mathrm{GPa}^{15}, 38 \mathrm{GPa}^{16}$, and $70.7 \mathrm{GPa}^{26}$. This wide range of LCP modulus values reflects the fact that the elastic modulus of an oriented LCP depends heavily on the processing conditions. Indeed, many researchers ${ }^{42-45}$ have found that the elastic modulus of extruded Vectra $A$ and $B$ rods increased dramatically with increasing draw ratio.

We have proposed a model to predict the elastic modulus of in situ composite strands with fibrillar LCP morphologies ${ }^{6,46}$. Our approach took into account both the change in aspect ratio of the reinforcing LCP phase and the variation of the orientation of the LCP chains during processing. We related the elastic moduli of the in situ composites to the LCP concentration and draw ratio through the well known Halpin-Tsai equation and a composite model of the reinforcing LCP phase. We envisaged the LCP phase itself as a composite of a perfectly oriented chain aggregate and a randomly oriented chain aggregate which are connected in series (see Figure 10). With increasing draw ratio, the fraction of oriented chains increases at the expense of random chains. Our results showed that the theoretical predictions agreed fairly well with the experimental results of the longitudinal elastic modulus of polycarbonate/Vectra in situ composite strands.

In this paper, we adopt the aggregate model first proposed by Crawford and $\mathrm{Kolsky}^{47}$, and further developed by Ward ${ }^{48}$ and Northolt and van der Hout ${ }^{49}$ to account for the change of the elastic modulus of the reinforcing LCP fibrils with drawing. Both of the models proposed by Ward ${ }^{48}$, and Northolt and van der Hout ${ }^{49}$ considered an oriented fibre as an assembly of polymer chain aggregates which are connected in series. They further assumed a uniform stress distribution throughout the oriented fibre. An external stress causes stretching of the aggregates as well as rotation of the aggregates due to misalignment (Figure 11). Here, we use the modified aggregate model proposed by Northolt and van der Hout $^{49}$ for the oriented LCP phase.

Northolt and van der Hout ${ }^{49}$ considered an oriented LCP fibre as being built up of a parallel array of identical fibrils. Each fibril consists of a series of end-toend connected aggregates of parallel aligned polymer chains. The orientation of the polymer chains (and the aggregates) is characterized by the angle between the chain axis (or the aggregate symmetry axis) and the fibre axis $(\theta)$. An external tensile stress, $\sigma$, applied along the fibre axis causes both an elongation of the aggregates and a rotation of the aggregates towards the fibre axis, as a result of shear deformation. Therefore, the total strain of the fibre is the sum of the strains due to the elongation and the shear deformation. If the polymer has a chain modulus $E_{\mathrm{c}}$, and a shear modulus $G$ along the chain direction, the tensile strain of the fibre $\left(\varepsilon_{\mathrm{f}}\right)$ can be derived as follows:

$$
\varepsilon_{\mathrm{f}}=\frac{\sigma}{E_{\mathrm{c}}}+\frac{\left\langle\sin ^{2} \theta\right\rangle}{2}[1-\exp (-\sigma / G)]
$$

where $\left\langle\sin ^{2} \theta\right\rangle$ represents the average orientation of the polymer chains with respect to the fibre axis. At small

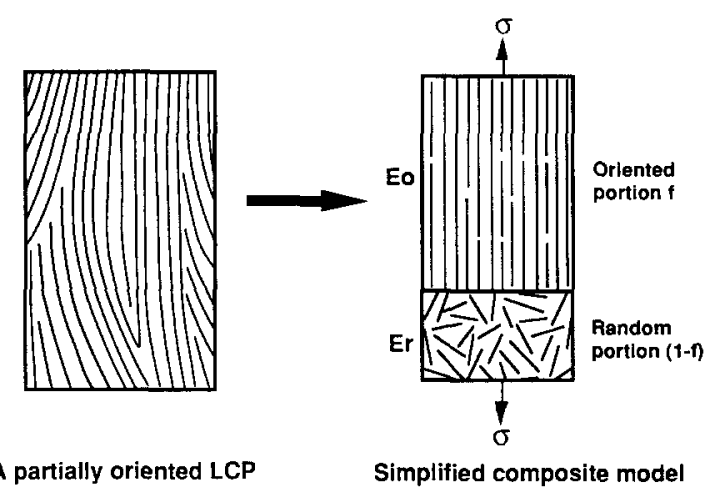

Figure 10 Composite model of an oriented LCP

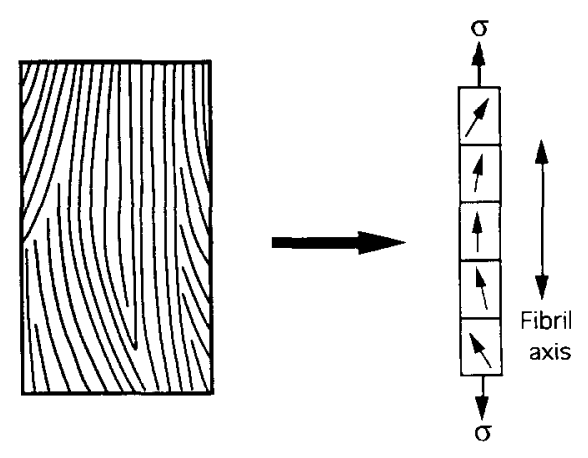

A partially oriented LCP

Aggregate Model

Figure 11 Aggregate model of an oriented LCP (after Northolt and Sikkema ${ }^{50}$ ) 


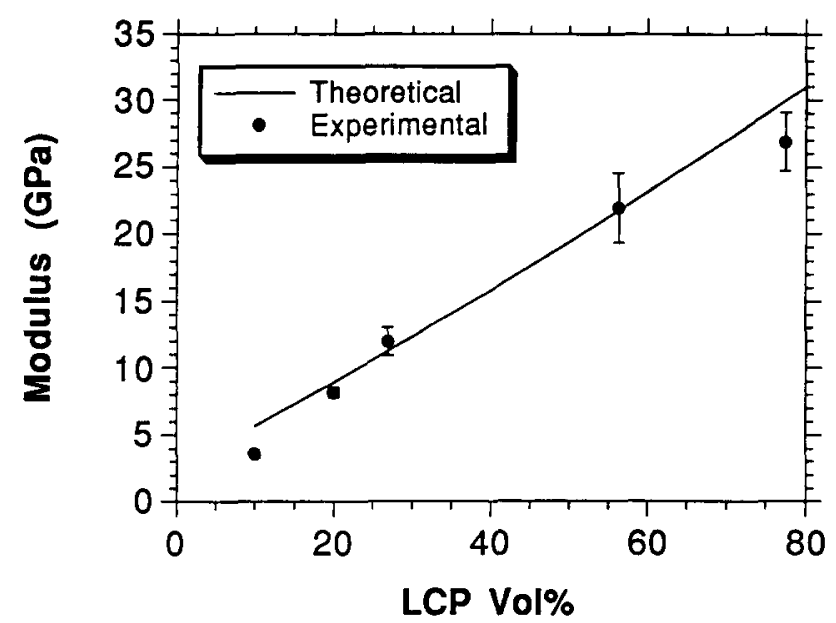

Figure 12 Comparison of theoretical predictions and experimental results for the variation of elastic modulus with LCP content of PC/LCP in situ composites with a draw ratio of 11.5

deformations, a simple relationship for the elastic modulus of the fibre, $E_{\mathrm{f}}$, can be obtained from equation (1):

$$
\frac{1}{E_{\mathrm{f}}}=\frac{1}{E_{\mathrm{c}}}+\frac{\left\langle\sin ^{2} \theta\right\rangle}{2 G}
$$

The equations derived by Ward and others differ from the above equation in the pre-factor of the second term $^{51.52}$, which results from the difference in the proposed shape of the aggregates. Ward ${ }^{51.53}$ adopted cube-shaped aggregates, while Northolt and van der Hout $^{49}$ used oblong-shaped aggregates. The aggregate models have been successfully applied to many oriented polymer systems ${ }^{49,54}$, including liquid crystalline polymers ${ }^{49-51,54-58}$. However, in most cases, $\left\langle\sin ^{2} \theta\right\rangle$ was used as a fitting parameter, but no correlation was obtained between $\left\langle\sin ^{2} \theta\right\rangle$ and the processing parameters. In this paper, we have attempted to relate the $\left\langle\sin ^{2} \theta\right\rangle$ parameter to the processing parameters, specifically the draw ratio $\lambda$, by using the so called floating rods model ${ }^{5,46}$.

According to the floating rods model, the order parameter $(f)$ of LCP chains with a draw ratio of $\lambda$ can be approximated as follows ${ }^{46}$.

$$
\begin{aligned}
f & =\frac{1}{2}\left(3\left\langle\cos ^{2} \theta\right\rangle-1\right) \\
& =\frac{2 \lambda^{3}+1}{2\left(\lambda^{3}-1\right)}-\frac{3}{2}\left[\frac{\lambda^{3}}{\left(\lambda^{3}-1\right)^{3 / 2}}\right] \sin ^{-1}\left(\frac{\lambda^{3}-1}{\lambda^{3}}\right)^{1 / 2}
\end{aligned}
$$

We therefore have:

$$
\left\langle\sin ^{2} \theta\right\rangle=-\frac{1}{\lambda^{3}-1}+\frac{\lambda^{3}}{\left(\lambda^{3}-1\right)^{3 / 2}} \sin ^{-1}\left(\frac{\lambda^{3}-1}{\lambda^{3}}\right)^{1 / 2}
$$

By inserting equation (4) into equation (2), we can relate the elastic modulus of the reinforcing LCP fibrils to the draw ratio of the composite by the following:

$$
\frac{1}{E_{\mathrm{f}}}=\frac{1}{E_{\mathrm{c}}}+\frac{1}{2 G}\left[-\frac{1}{\lambda^{3}-1}+\frac{\lambda^{3}}{\left(\lambda^{3}-1\right)^{3 / 2}} \sin ^{-1}\left(\frac{\lambda^{3}-1}{\lambda^{3}}\right)^{1 / 2}\right]
$$

In a previous paper ${ }^{46}$, we showed that the longitudinal elastic modulus $\left(E_{1}\right)$ of in situ composite strands can be written as follows:

$$
E_{\mathrm{l}}=E_{\mathrm{m}}\left(\frac{1+2 \lambda^{3 / 2} \eta V_{\mathrm{f}}}{1-\eta V_{\mathrm{f}}}\right) \quad \text { with } \eta=\frac{E_{\mathrm{f}} / E_{\mathrm{m}}-1}{E_{\mathrm{f}} / E_{\mathrm{m}}+2 \lambda^{3 / 2}}
$$

where $E_{\mathrm{f}}$ and $E_{\mathrm{m}}$ are the elastic moduli of the reinforcing fibrous LCP phase and the matrix, respectively. $\lambda$ is the draw ratio of the composite fibre and $V_{\mathrm{f}}$ is the volume fraction of the fibrous LCP phase.

As in our previous paper, we again use $E_{\mathrm{m}}=2.5 \mathrm{GPa}$ for the modulus of the polycarbonate matrix and $E_{\mathrm{c}}=110 \mathrm{GPa}$ for the chain modulus of the Vectra liquid crystalline polymer ${ }^{46}$. The shear modulus $(G)$ of the Vectra liquid crystalline polymer was measured by Zhang et al. ${ }^{57}$ to be $1.2 \mathrm{GPa}$ at room temperature. This values falls well within the reported shear moduli values for Vectra liquid crystalline polymers, such as $0.9 \mathrm{GPa}^{56}$, 2.0 $\mathrm{GPa}^{51}, 1.7 \mathrm{GPa}^{58}$, and $1.47 \mathrm{GPa}^{59}$. With these values, the longitudinal elastic modulus of the in situ composites can be calculated as a function of the LCP content and the draw ratio by using equations (5) and (6).

Comparisons of the theoretical predictions with the experimental results are shown in Figures 12-14. Again, we see a fairly good correspondence between the theoretical values and the experimental ones. This good

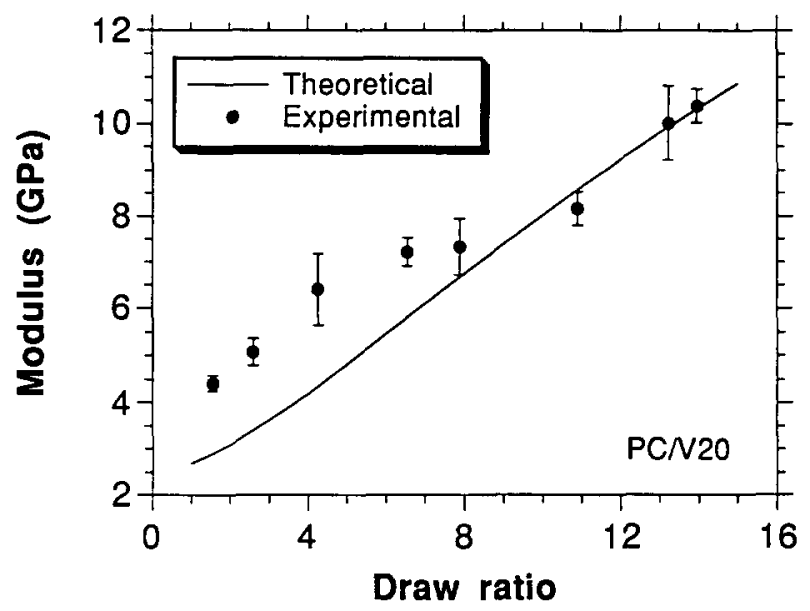

Figure 13 Comparison of theoretical predictions and experimental results for the variation of elastic modulus with draw ratio of $\mathrm{PC} / \mathrm{LCP}$ in situ composites with 20 vol\% LCP

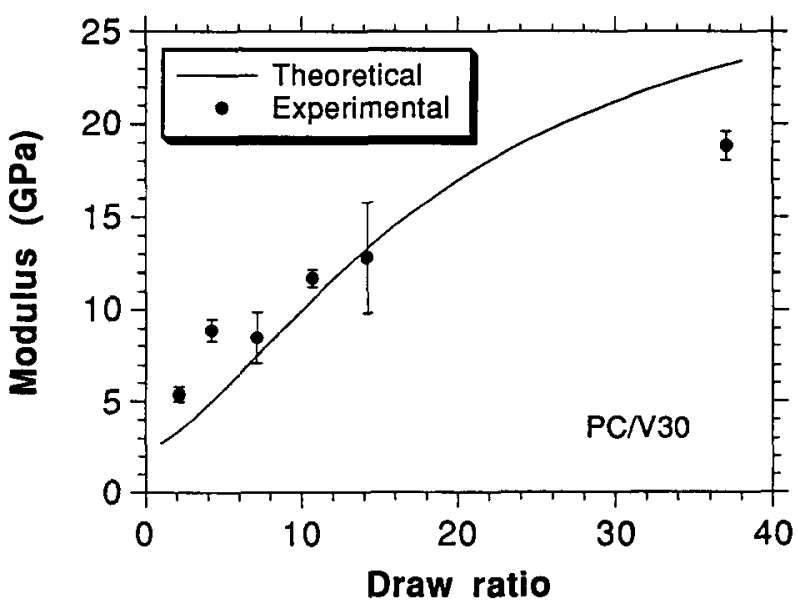

Figure 14 Comparison of theoretical predictions and experimental results for the variation of elastic modulus with draw ratio of $\mathrm{PC} / \mathrm{LCP}$ in situ composites with 26.9 vol\% LCP 
agreement between theory and experiment once again corroborates our previous conclusion that the elastic modulus of in situ composites is dominated by the aspect ratio of the reinforcing LCP phase and the molecular orientation of the LCP chains ${ }^{5,7}$.

We can see that the performance of the present model is almost as good as the previous one ${ }^{46}$. This is not surprising in view of the many similarities between the two. First, both models are based on the Halpin-Tsai equation, and use the affine deformation scheme to derive the aspect ratio for the LCP phase. Furthermore, both models conceive the oriented LCP phase as aggregates of LCP chains, and propose a series connection of the chain aggregates. Thirdly, in both models, the orientation of the LCP chains is approximated by the affine deformation of the rigid-rod molecules. In fact, equation (6) in this paper is exactly the same as equation (5) in ref. 46. This present model differs from the previous one in that Northolt's aggregate model is used for the reinforcing LCP phase instead of the composite model. According to Northolt's model, an external tensile stress causes both the stretching, and the rotation of the chain aggregates due to misorientation, while in the composite model, the external stress causes only the stretching of both the perfectly oriented chain aggregate and the randomly oriented chain aggregate. The LCP chain misorientation is taken into account by changing the volume ratio of the two aggregates.

\section{CONCLUSIONS}

The mechanical properties of in situ composites based on blends of polycarbonate and a liquid crystalline copoly(ester amide) (Vectra B950) have been studied as functions of LCP concentration and draw ratio. It is found that both the elastic modulus and the tensile strength of the in situ composites increase steadily with the LCP concentration and the draw ratio, while the ultimate tensile strain, however, decreases with these two parameters. A model is proposed for the elastic modulus of the in situ composites, in which the elastic modulus of the LCP phase is related to the draw ratio via Northolt's model for the LCP phase. Equations for the longitudinal elastic modulus of the in situ composites are then derived based on the Halpin-Tsai equation. The experimental elastic moduli of the in situ composites are found to agree fairly well with the theoretical values.

\section{REFERENCES}

Kiss, G. Polym. Eng. Sci. 1987, 27, 410

Siegmann, A., Dagan, A. and Kenig, S. Polymer 1985, 26, 1325

Bassett, B. R. and Yee, A. F. Polym. Compos. 1990, 11, 10

Amundsen, R. J. and Yee, A. F. in 'Proceedings of the American Society for Composites', 5th Technical Conference E. Lansing. MI, June 1990, p. 209

5 Lin. Q. and Yee, A. F. Am. Chem. Soc. Div. Polym. Chem. Polym Prepr. 1992, 33(1), 298

6 Lin, Q. and Yee, A. F. Am. Chem. Soc. Div. Polym. Chem. Polym. Prepr. 1993, 34(1), 753

7 Lin, Q., Jho, J. Y. and Yee, A. F. Polym. Eng. Sci. 1993, 33, 789

8 Blizard, K. G., Federici, C., Federico, O. and Chapoy, L. L. Polim. Eng. Sci. 1990, 30, 1442

9 Dutta, D., Weiss, R. A. and Kristal, K. Polym. Compos. 1992. 13. 394
10 Sun, T., Baird, D. G., Hung, H. H., Done, D. S. and Wilkes, G. L. J. Compos. Mater. 1991, 25, 788

Jung, S. H. and Kim. S. C. Polym. J. 1988, 20, 73

Datta, A., Chen, H. H. and Baird, D. G. Polymer 1993, 34, 759

Crevecoeur, G. and Groeninckx, G. Polym. Eng. Sci. 1990, 30, 532 Beery, D., Kenig, S. and Siegmann, A. Polym. Eng. Sci. 1991, 31, 459

15 Kohli, A., Chung, N. and Weiss, R. A. Polym. Eng. Sci. 1989 , 29. 573

16 Isayev, A. I. and Modic, M. J. ANTEC 1986, 573

17 Zhuang, P., Kyu, T. and White, J. L. Polym. Eng. Sci. 1988, 28 1095

18 Shin, B. Y. and Chung, I. J. Polym. J. 1989, 21, 851

19 Kimoto, M. and Hiraguchi, T. Kobunshi Ronbunshu 1992, 49, 105

20 Nobile, M. R., Amendola, E.. Nicolais, L., Acierno, D. and Carfagna, C. Polym. Eng. Sci. 1989. 29, 244

21 Valenza, A., La Mantia, F. P., Paci, M. and Magagnini, P. L. Int. Polym. Process. 1991, 6, 247

22 Chapleau. N., Carreau. P. J., Peleteiro, C., Lavoie, P.-A. and Malik. T. M. Polym. Eng. Sci. 1992, 32, 1876

23 Ogata, N., Tanaka, T., Ogihara, T., Koshida, K., Kondou, Y. Hayashi, K. and Yoshida, N. J. Appl. Polym. Sci. 1993, 48, 383

24 La Mantia, F. P. and Valenza, A. Makromol. Chem. Macromol Simp. 1990, 38, 183

25 Shin, B. Y, and Chung, I. J. Polym. Eng. Sci. 1990, 30, 22

26 Li, J. X.. Silverstein, M. S., Hiltner, A. and Baer, E. J. Appl. Polym Sci. 1992, 44, 1531

27 Seppala, J., Heino, M. and Kapanen. C. J. Appl. Polym. Sci. 1992, 44, 1051

28 Shin, B. Y. and Chung, I. J. Polym. Eng. Sci. 1990, 30, 13

29 Shin, B. Y., Jang, S. H., Chung. I. J. and Kim, B. S. Polym. Eng. Sci. $1992,32,73$

30 Amano, M. and Nakagawa, K. Polymer 1987, 28, 263

31 Qin, Y., Brydon, D. L., Mather, R. R. and Wardman. R. H. Polymer 1993, 34, 1196

32 Carfagna, C., Amendola, E., Nicolais, L., Acierno, D. Francescangeli, O., Yang, B. and Rustichelli, F. J. Appl. Polym. Sci. $1991, \mathbf{4 3}, 839$ Isayev, A I and Subramanian, P R. Polym Eng Sci. 1992,32,85 Kulichikhin, V. G., Vasil'eva, O. V., Litvinov, I. A. Antipov, E. M., Parsamyan, I. L. and Plate, N. A. J. Appl. Polym. Sci. 1991, 42, 363

35 Subramanian, P. R. and Isayev, A. I. Polymer 1991, 32, 1961

36 Zaldua, A., Munoz, M. E., Pena, J. J. and Santamaria, A. Polymer $1992,33,2007$

37 Crevecoeur, G. and Groeninckx, G. Polym. Compos. 1992, 13, 244 Bretas, R. E. S. and Baird, D. G. Polymer 1992, 33, 5233 Ajji, A., Brisson, J. and Qu, Y. J. Polym. Sci., Polym. Phys. Edn 1992, 30, 505

. A J Appl Polym. Sci. 1993,48, 1677 Lin, Q. and Yee, A. F. unpublished results, 1993

Chung, T. S. J. Polym. Sci., Polym. Phys. Edn 1986, 26, 1549

Kenig, S. Polym. Eng. Sci. 1987, 27.887

Kenig, S. Polym. Eng. Sci. 1989, 29, 1136

Turek, D. E. and Simon, G. P. Polymer 1993, 34, 2750

Lin, Q. and Yee, A. F. Polym. Compos. 1994, 15, 156

Crawford, S. M. and Kolsky, H. Proc. Phy's. Soc. 1951, B64, 119

Ward, I. M. Proc. Phys. Soc. 1962, 80, 1176

Northolt, M. G. and van der Hout, R. Polymer 1985, 26, 310 Northolt, M. G. and Sikkema, D. J. in 'Liquid Crystal Polymers: From Structures to Applications' (Ed. A. A. Collyer), Elsevier. London, 1992, p. 273

51 Green, D. I., Unwin, A. P.. Davies, G. R. and Ward, I. M Polymer $1990,31,579$

52 Allen, S. R. and Poche, E. J. Polymer 1990, 31, 996

53 Ward, I. M. 'Mechanical Properties of Solid Polymers', Wiley, Chichester, 1983, Ch. 10 , p. 247

54 Northolt, M. G. Polymer 1980, 21, 1199

55 Davies, G. R. and Ward, I. M. in 'High Modulus Polymers' (Eds A. E. Zachariades and R. S. Porter), Marcel Dekker, New York, 1988 , p. 37

56 Troughton, M. J., Davies, G. R. and Ward, I. M. Polymer 1989 30, 58

57 Zhang, H., Davies, G. R. and Ward, I. M. Polymer 1992, 33, 2651

58 Turek, D. E. and Simon, G. P. Polymer 1993, 34, 2763

59
Choy, C. L.. Leung, W. P. and Yee, A. F. Polymer 1992, 33, 1788 